\title{
Potential implication of the COVID-19 pandemic on altitude training programming
}

\author{
G Manferdelli1,2, DJ Bishop3, M Franchi4, F Sarto4, O Girard5, S Porcelli6,7
}

1 Institute of Biomedical Technologies, National Research Council, Segrate, Italy

${ }_{2}$ School of Health and Exercise Sciences, University of the West of Scotland, UK

3 Institute for Health and Sport (IHES), Victoria University, Melbourne, Australia

4 Institute of Physiology, Department of Biomedical Sciences University of Padua, Padua, Italy

5 School of Human Sciences, Exercise and Sport Science, University of Western Australia, Perth, WA, Australia

${ }_{6}$ Department of Molecular Medicine, University of Pavia, Italy

${ }_{7}$ Medical Committee, Italian Winter Sports Federation, Milan, Italy

Please cite as: Manferdelli, G., et al. 2020 Potential implication of the COVID-19 pandemic on

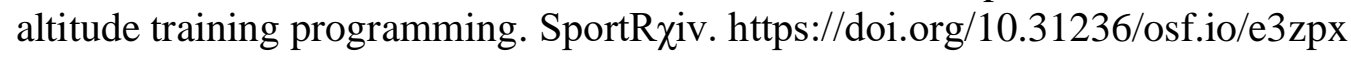

\section{KEYWORDS:}

Altitude; Sports medicine; Elite performance; Exercise training; Prevention

Correspondent Author:

Simone Porcelli, MD, PhD

Department of Molecular Medicine

University of Pavia

Via Forlanini 14, 27100, Pavia, Italy

simone.porcelli@unipv.it 
Low-to-moderate altitude (2000-2500m) training camps are an integral part of many athletes' training programs.1 Despite potential positive effects on performance, sojourning at altitude represents an important stress on the human body, with transiently increased pulmonary and kidney stress, neuroendocrine dysregulation, and immune perturbations.2 This highlights the importance of careful planning and organisation, which may be more critical during the current COVID-19 (coronavirus disease 2019) pandemic.

Preliminary reports suggest that hypoxemia and inflammation induced by COVID-19 results in heterogenous lung injury and acute respiratory distress syndrome, eventually leading to acute respiratory failure cases.3 Even though COVID-19 is primarily a respiratory disease, it can also negatively affect the cardiovascular system, exposing affected patients to myocarditis or myocardial damage.4 Moreover, COVID-19 may impair renal function as well as the circulatory and the immune system.

As we move towards the gradual resumption of normal life once the COVID-19 emergency subsides, athletes will also seek to resume their regular training. With the cancellation or postponement of the almost half of the scheduled sporting events in 2020 (e.g., the Tokyo Olympics, many of the cycling tours, World Marathon Majors, and team-sport competitions around the world), many athletes will look to plan at least one altitude training camp in the next 12 to 18 months. 1 During these altitude training periods, athletes will be required to manage changes in training load and to stay socially close to other athletes. Moreover, athletes will be forced to cope with physiological adaptations to altitude, and hypoxia-induced stress on the pulmonary, cardiovascular, renal and immune systems that may be exacerbated by prior exposure to COVID-19. Altitude training may also lead to greater susceptibility to COVID-19 infection and its sequelae by increasing the level of hypoxemia and further depressing the immune function.5,6 Thus, specific recommendations for altitude training programming are needed to preserve athletes' health in this post COVID-19 environment. 
BEFORE GOING: In our opinion, each athlete should be tested for positivity to COVID-19, followed by a careful medical examination and a complete blood screening (white blood cells, haemoglobin, inflammation markers, iron status). In athletes previously infected with COVID-19 (i.e., returning a positive antibody test), medical examination should also evaluate cardiological, pulmonary and, in general, systemic sequelae of infection. COVID-19 positive athletes should not engage in any form of altitude training and should remain isolated for at least 14 days after symptoms cease. For athletes that don't test positive to infection and don't show contraindication to exercise training, we suggest assessing that cardiorespiratory and blood oxygen saturation responses to maximal exercise are normal, as previously recommended.1 The addition of exercise testing and/or training sessions with reduced fraction of oxygen may be useful to evaluate the individual physiological response to hypoxia before going to altitude.7

GOING TO ALTITUDE: During travelling to altitude camps, athletes should follow the guidelines provided by national health agencies (e.g. wearing masks, using gloves, social distancing). Moreover, a slower ascent profile to altitude may be suggested.

AT ALTITUDE: Athletes should be directed to reduce the time spent in close contact with their teammates during daily activities and training sessions. Workout should be accurately modified, reducing number of athletes involved (e.g., players in small sided games in team sports) and accurately managing training load. Gradual increase of volume/intensity is suggested in order to cope with inadequate training stimulus over the last months. Classic parameters related to physiological adaptations to altitude should be measured and recorded more frequently than usual during the day. Higher-than-normal changes in $\mathrm{SpO}_{2}$ and heart rate readings are clear signs of maladaptive response to acute exposure to hypoxia.1 Signs and symptoms related to COVID-19, such as elevated core temperature or loss of taste and/or smell, could be added to the daily screening. Athletes reporting 
abnormal values in reference to previous altitude camps should immediately isolate themselves from others and, after medical evaluation, moved to a lower elevation (or supplemented with oxygen if needed) and return to near sea level if symptoms persist. As suggested by others, 8 in the event of minor respiratory illness the "neck check" rule should not be followed. That is, athletes should not continue to exercise even if their symptoms and clinical signs are only confined to the upper airways; a more prolonged rest period is recommended until these symptoms subside.

RETURN FROM ALTITUDE: Following return to sea level, a careful monitoring of an athlete's health, especially the immune system and signs/symptoms related to COVID-19, is advised. A progressive reintegration before starting to train with other athletes is also suggested.

In conclusion, in the context of the COVID-19 global health emergency, we suggest adopting specific caution before, during and in return from altitude exposure. In particular, early symptoms of maladaptation to hypoxia, and respiratory problems should be considered with attention as they can mask COVID-19 symptoms. 
Authors' contribution: GM and SP conceived the article; GM, DJB, MF, FS, OG, and SP contributed in writing the article; all authors have commented, revised and approved the article in its final form before submission.

Conflict of Interests Disclosure: The authors would like to declare the presence of no conflict of interest.

Funding: The authors have not declared a specific grant for this research from any funding agency in the public, commercial or not-for-profit sectors. 


\section{References}

1 Mujika I, Sharma AP, Stellingwerff T. Contemporary Periodization of Altitude Training for Elite Endurance Athletes: A Narrative Review. Sport Med 2019;49:1651-69. doi:10.1007/s40279-019-01165-y

2 Mazzeo RS. Altitude, exercise and immune function. Exerc Immunol Rev 2005;11:6-16.

3 Wang T, Du Z, Zhu F, et al. Comorbidities and multi-organ injuries in the treatment of COVID-19. Lancet 2020;395:e52. doi:10.1016/S0140-6736(20)30558-4

4 Madjid M, Safavi-Naeini P, Solomon SD, et al. Potential Effects of Coronaviruses on the Cardiovascular System: A Review. JAMA Cardiol 2020;10:1-10. doi:10.1001/jamacardio.2020.1286

5 Bergeron MF, Bahr R, Bärtsch P, et al. International Olympic Committee consensus statement on thermoregulatory and altitude challenges for high-level athletes. Br J Sports Med 2012;46:770-9. doi:10.1136/bjsports-2012-091296

6 Girard O, Amann M, Aughey R, et al. Position statement-Altitude training for improving team-Sport players' performance: Current knowledge and unresolved issues. Br J Sports Med 2013;47. doi:10.1136/bjsports-2013-093109

7 Pla R, Brocherie F, Le Garrec S, Richalet J-P. Effectiveness of the hypoxic exercise test to predict altitude illness and performance at moderate altitude in high-level swimmers. Physiol Rep . 2020;8:e14390. https://doi.org/10.14814/ phy2.14390

8 Hull JH, Loosemore M, Schwellnus M. Respiratory health in athletes: facing the COVID-19 challenge. Lancet Respir Med 2020;0:2019-20. doi:10.1016/S2213-2600(20)30175-2 